\title{
О генетическом методе
}

\author{
В. И. ШАЛАК
}

\begin{abstract}
The aim of this paper is to answer the question what is the genetic method for constructing theories. The distinctive feature of genetic theories is the use of functional languages, and proof in these theories is essentially based on the structure of terms.

Ключевые слова: генетический метод, генетическое рассуждение, геометрия Евклида, рекурсивная арифметика, функциональный язык, реляционный язык
\end{abstract}

\section{1 Введение}

Время от времени в логической литературе можно встретить упоминания о генетическом стиле мышления, генетических способах рассуждений, генетической дедукции и о генетическом методе построения теорий в его противопоставлении аксиоматическому $[1,3,6,7,10,11]$. При этом авторы, как правило, ограничиваются лишь примерами, которые, по их мнению, должны быть отнесены к генетическим, а также перечисляют возможные отличительные признаки, но не формулируют сути генетического подхода столь же ясно и строго, как это было сделано, например, в отношении аксиоматического. Вопрос о том, что же такое генетические способы рассуждений и что такое генетический метод построения теорий, остается без ответа.

Большинство авторов сходятся во мнении, что исторически первой теорией, построенной генетическим методом, была геометрия Евклида $[9,13]$. Она занимает в истории науки уникальное место, поскольку долгое время была одним из наиболее почитаемых разделов математики и служила едва ли не идеальным образцом для подражания при построении научных теорий. Курьез, связанный с геометрией Евклида, заключается в том, что, несмотря на общее убеждение в ее дедуктивном характеpe, до сих пор нет ясного понимания, в чем его специфика и 
на каких теоретических принципах основывалась эта дедукция. Дело в том, что во время написания «Начал» Евклида и вплоть до XIX в. существовала единственная созданная Аристотелем законченная логическая теория, язык которой был слишком беден, чтобы в нем могли быть представлены сложные математические понятия и доказательства теорем геометрии. Сказанное относится не только к геометрии, но и к другим математическим дисциплинам, которые более двух тысяч лет развивались без видимой опоры на логику.

\section{2 Геометрия Евклида}

В 1899 году Д. Гильбертом была предложена логически строгая аксиоматизация геометрии [2], которая, однако, сильно отличалась от своей прародительницы как по набору исходных понятий, так и по виду аксиом.

Так, например, если у Евклида первый постулат геометрии сформулирован следующим образом:

«Допустим:

1. Что от всякой точки до всякой точки <можно> провести линию» [9, с. 14],

то Д. Гильберт передает его посредством двух аксиом:

«I1. Для любъх двух точек A, В существует прямая a, принадлежащая каждой из этих двух точек $A, B$.

I2. Для двух точек $A, B$ существует не более одной прямой, принадлежащей каждой из точек $A, B »[2$, c. 57].

Если Евклид говорит о возможности через две точки провести линию, то у Д. Гильберта речь идет об отношении принадлежности между уже существующими линиями и точками. Уже этого примера достаточно, чтобы заметить принципиальное отличие двух подходов к построению геометрии.

С подробным анализом «Начал» Евклида можно ознакомиться в комментариях к ним Д.Д. Мордухая-Болтовского [8]. Он, в частности, пишет: 
«Только отказавшись от проектирования в прошлое современных формально-логических понятий, мы будем в состоянии понять, что представляли для самого Евклида постулаты. Евклид вовсе не приписъвал идеального существования геометрическим обоектам, как это делал Платон. При доказательстве какой-нибудв теоремы производилось построение нужной геометрической фигуры, которая, таким образом, и вызывалась к существованию. Возможность существования прямой, круга и т.д. обусловливалась признанием возможности производящего их акта, мы сказали бы - построения. Более того, признание возможности этого акта вынуждало $\kappa$ признанию некоторых истин; так, признавая согласно постулату 3 возможсность описывания кругов, мы тем самым приходили к признанию необходимости пересечения кругов, проходящих через иентры друг друга. [...] При таком понимании геометрия перестает быть чисто логчческой дисииплиной: линейка и ииркуль с дозволенными операчиями служат для убеждения так же, как и силлогизм» $[8$, c. 238-241].

Попробуем понять, почему именно такой подход к построению геометрии был избран Евклидом.

$\mathrm{C}$ нашей точки зрения, причины этого следует искать в общих философских представлениях об устройстве окружающего мира и языке как конечном средстве объективации знаний. Мир представлялся состоящим из вещей и свойств, которыми эти вещи могут обладать. Познание было возможно, если законы мысли следовали законам бытия. Субъектно-предикатная структура предложений естественного языка, по мысли древних философов, отражала объективно существующие связи между вещами и свойствами. В то же время отношения, даже такие простые, как «x больше $y$ », не обладали объективным существованием. С этой проблемой сталкивается Сократ в диалоге Платона «Теэтет».

И значит, если бы тебе сказали, что один человек головою больше другого, а другой головою менъше, 
ты не принял бы этого утверждения, но решительно его отклонил, заявивши так: „Я могу сказать лишь одно - что всякая вещь, которая больше другой вещи, такова лишь благодаря большому, то есть она становится больше благодаря большому, а менъшее становится меньшим лишь благодаря малому, то есть малое делает его менъшим“. А если бъ ты признал, что один человек головою больше, а другой меньше, тебе пришлось бъ, я думаю, опасаться, как би не встретить возражения: прежде всего в том, что большее, у тебя есть большее, а менъшее - меньшее по одной и той же причине, а затем и в том, что большее делает бо́льшим малое, - ведь голова-то мала! [Платон, Теэтет, 100е-101a].

В приведенной цитате обращает на себя внимание следующее. Сократ видит проблему в приписывании предиката бытъ бо́льшим, но не испытывает трудностей в употреблении этого же термина в смысле результата сравнения вещей. Возможно, это и есть ключ к пониманию Евклида.

Если объективное существование отношений не вписывалось в онтологию вещей и свойств, то возможность совершать действия с вещами, создавать из них более сложные конструкции, производить измерения, не противоречила этой онтологии. Можно было смешать вино с водой и получить разбавленное вино. Можно было сосчитать корабли в порту, измерить высоту храма. Можно было соединить две точки прямой линией и получить отрезок. Но сказать, что две точки и линия находятся в отношении принадлежности, было бессмыслицей.

Чтобы получить сложный подлежащий исследованию геометрический объект, требовалось възвать его $к$ существованию путем построения. По Евклиду, набор элементарных действий, используемых для построения геометрических объектов, невелик и перечислен в трех постулатах 1, 2 и 3:

«Допустим:

1. Что от всякой точки до всякой точки <можно> провести линию.

2. И что ограниченную прямую <можно > непрерьвно продолэсать до прямой. 
3. И что из всякого чентра и всяким раствором <может быть > описан круг.

4. И что все прямые угль равны между собой.

5. И если прямая, падающая на две прямве, образует внутренние и по одну сторону углы, меньшие двух прямых, то продолюенные эти две прямые неограниченно встретятся с той стороны, где углы менъшие двух прямых» $[9$, с. 14-15].

Четвертый постулат часто относят к аксиомам, поскольку он не говорит о способе построения, а содержит утверждение о равенстве прямых углов. Знаменитый пятый постулат является инструкцией по нахождению точки пересечения двух линий. Он не содержит указания, где именно пересекутся эти линии, но призывает продолжать их до нахождения требуемой точки. Сегодня мы уже знаем, как вычислить расстояние, на котором линии пересекутся.

Силлогистика Аристотеля - это теория отношений в сфере общих терминов. В то время еще не существовало понятия функции, и потому оно не могло быть включено в логику. Но ничто не мешало говорить и рассуждать о результатах выполнения тех или иных действий, коль скоро эти результаты также были вещами.

\section{3 Объекты генетических теорий}

Многие исследователи подчеркивают, что одной из отличительных особенностей генетического подхода к построению теорий является «способ введения их объектов» [10, с. 422]. Если посмотреть на структуру доказательств Евклида, то начинаются они либо с подробной демонстрации того, каким образом строится объект, подлежащий дальнейшему исследованию, либо со ссылок на ранее построенные объекты. В современной логике пошаговому построению геометрических объектов соответствует композициональная структура термов. Отношение чертежа к соответствующему идеальному геометрическому объекту то же, что и отношение знака к означаемому. Чертежи, создаваемые путем ограниченного набора доступных операций, играли не только иллюстративную роль, но и роль формального языка геометрии. Чертеж выступал в роли терма. В отличие от 
современных линейных языков язык геометрии был принципиально двумерным. Его недостатком было лишь отсутствие информации об очередности построения, поскольку один и тот же геометрический объект мог быть получен различными способами. Поэтому, во избежание двусмысленности, геометрический чертеж с необходимостью должен был сопровождаться словесными комментариями о выполненных действиях. Дальнейшие рассуждения о свойствах объекта существенным образом опирались именно на способ его построения (структуру терма). Чертеж (терм) должен был оставаться перед глазами на протяжении всего рассуждения. Иногда в ходе доказательства требовалось дополнить чертеж новыми деталями (построить новый терм). Способ введения объектов геометрии Евклида был исторически обусловлен. Но отсюда вовсе не следует, что он был плох. Наглядная структура термов геометрии значительно облегчала дальнейшие рассуждения и даже позволяла обходиться без принятия дополнительных аксиом, необходимость которых была осознана значительно позже.

Еще одним примером теории, объекты которой вводятся генетически, является арифметика. Натуральные числа получаются путем последовательного прибавления единицы к числу ноль. В языке арифметики их задают посредством индуктивных определений и представляют в виде потенциально бесконечной последовательности термов $<0, S(0), S(S(0)), S(S(S(0))), \ldots>$. Операции сложения и умножения определяются рекурсивно путем сведения сложных термов к более простым. Благодаря этому структура арифметических термов однозначно кодирует способ их вычисления.

- $x+0=x$

- $x+S(y)=S(x+y)$

- $x \times 0=0$

- $x \times S(y)=(x \times y)+x$

Кроме логического отношения равенства никакие другие отношения в арифметике не являются необходимыми, их всегда 
можно ввести посредством определений. При этом следует отметить, что удобная символика для записи арифметических выражений существовала не всегда, и долгое время, как и в случае с геометрией, ученые были вынуждены обходиться громоздкими словесными описаниями. Но они все-таки могли проводить рассуждения и доказывать сложные теоремы.

Если сравнить способы введения объектов в двух рассмотренных выше теориях, то обнаружится, что в геометрии, в отличие от арифметики, индуктивные определения не используются. В арифметике все числа порождаются путем прибавления единицы к постулируемому существующим единственному числу 0 , а в геометрии точки, к которым также могут применяться операции, предполагаются уже существующими. В результате множество натуральных чисел оказывается счетным, а множество точек и соответственно множество идеальных объектов геометрии - континуальны.

\section{4 Логическая техника}

В качестве второго отличия генетических теорий от аксиоматических указывают на используемую «логическую технику» $[10$, c. 422 .

«... в качестве логических средств для развития генетической теории достаточно индуктивных определений и процессов, формализуемых в теории рекурсивных функиий» [10, с. 430].

В подтверждение этой точки зрения можно было бы привести книгу Р. Гудстейна, где примитивно рекурсивная арифметика формализована в виде исчисления равенств [5, С. 185]. Каждая теорема данного исчисления - это утверждение о равенстве двух арифметических термов. Английский оригинал книги Р. Гудстейна имеет подзаголовок «A development of recursive arithmetic in a logic-free equation calculus», который в русскоязычном издании был не совсем точно переведен как «Развитие рекурсивной арифметики в исчислении равенств, свободном от логических связок».

В эквациональном исчислении Р. Гудстейна доказательный переход от одного выражения к другому обосновывается исключительно рекурсивными определениями функций (структурой 
термов, представляющих эти функции) и свойствами отношения равенства, которое «по содержанию соответствует чемуто такому, что в известном смысле предшествует определению какого-бъ то ни было предиката, а именно - возможности различения элементов индивидной области» [4, с. 209]. Bсе правила вывода - прямые.

При всей стройности и красоте таких теорий все-таки не следует отождествлять используемую в них логическую технику с генетическим стилем мышления. Рекурсивный стиль мышления является лишь частным случаем генетического.

Обратимся еще раз к Евклиду. Кроме постулатов, в его геометрии есть и аксиомы.

«1. Равные одному и тому же равны и между собой.

2. И если к равным прибавляются равные, то и иелье будут равны.

3. И если от равных отнимаются равные, то остатки будут равны.

4. И если к неравным прибавляются равные, то иелье будут не равны.

5. И удвоенные одного и того же равны между собой.

6. И половины одного и того же равны между собой.

7. И совмещающиеся друг с другом равны между собой.

8. И иелое больие части.

9. И две прямые не содержат пространства» [9, с. 15].

Для наглядной записи первых шести аксиом воспользуемся символом отношения равенства «=»и функциональными символами «..+..», «..-..», «.. $. . . », \ll . . / . . »$.

Первая аксиома - это транзитивность и симметричность отношения равенства.

1. Если $a=c u b=c$, mo $a=b$.

Следующие пять аксиом говорят о конгруэнтности равенства.

2. Если $a=b$ u $c=d$, mo $a+c=b+d$.

3. Если $a=b$ и $c=d$, то $a-c=b-d$. 
4. Если не-(a=b) ис $=d$, то не- $(a+c=b+d)$.

5. $Е с л и ~ a=b$, то $a \times 2=b \times 2$.

6. Если $a=b$, то $a / 2=b / 2$.

Седьмая аксиома определяет один из способов вычисления характеристической функции отношения равенства между геометрическими объектами. Два объекта равны (конгруэнтны), если их можно совместить друг с другом.

Аксиомы нужны Евклиду для того, чтобы доказывать свойства геометрических объектов. Рассуждая, он использует не только прямые правила, но и косвенные. Так, например, уже в шестом предложении первой книги «Начал» [9, с. 20-21], содержащем утверждение, что если углы в основании треугольника равны, то равны и прилегающие к ним стороны, Евклид прибегает к рассуждению от противного, приходит к противоречию с восьмой аксиомой и отсюда заключает, что стороны равны. Как и положено в рассуждении от противного, он вынужден использовать снятие двойного отрицания: «Значит, $A B$ не будет не равной $A C$; значит, она ей равна» [9, с. 21]. В другом, более раннем, издании «Начал» снятие двойного отрицания передано словами: «Чего ради АВ не будет неравна АC; следственно равна» $[13$, с. 13$]$. Но закон снятия двойного отрицания не относится к числу законов конструктивной логики.

Единственное, что объединяет рассуждения в рекурсивной арифметике и в геометрии Евклида, - это опора на структуру термов, которая в случае арифметики представлена рекурсивными определениями функций, а в случае геометрии - очередностью выполнения операций при построении чертежей.

\section{5 Генетический метод в научной практике}

Генетический подход к построению и развертыванию теорий обнаруживается не только в математике, но и в других науках. На это обращает внимание В.С. Степин [11]. Он приводит ряд примеров из научной практики, которые наиболее адекватно описываются именно в терминах генетического стиля мышления.

«При анализе теоретических текстов обнаруживается, что даже в высокоразвитых теориях, широко использующих приемы формализованной аксиома- 
тики, кроме бормально-аксиоматической части существует некоторый принципиальный небормальный остаток, причем организованный вовсе не по нормам аксиоматико-дедуктивного построения.

Виясняется, что в процессе дедуктивного развертывания теории наряду с аксиоматическими приемами рассуждения большую роль играет генетически-конструктивный метод построения знаний, причем выступающий в форме своего содержательного варианта» [11, с. 127].

Законы классической механики содержат самое общее описание механического взаимодействия тел, представленных как материальные точки. Их можно применить и для анализа осцилляторов, но прежде необходимо выявить все элементы исследуемой модели и уточнить характер взаимодействия между ними. Лишь после этого с использованием законов механики можно получить уравнение малых колебаний.

«В рассуждениях физика осииллятор играет примерно ту же роль, что и геометрическая фигура в рассуждениях математика. Он позволяет установить связь между силой и величиной отклонения материальной точки от положения равновесия, что, в свою очередъ, приводит к конкретизации второго закона Ньютона и превращению его в уравнение колебаний» [11, с. 129-130].

Осцилляторную модель можно описать и с помощью реляционного языка, но для этого она уже должна быть представлена в виде некоторого мысленного конструкта. Поэтому более естественным способом описания модели является ее представление в языке именно в виде конструкта по аналогии с тем, как это делается в геометрии. После этого следует изучение характера взаимодействия элементов модели (структуры терма) и на основе этого делаются заключения о ее свойствах. Такой способ рассуждений, от мысленного конструкта к его свойствам, характерен для многих естественных наук.

«Развертывание знаний осуществляется в этом случае путем мысленного экспериментирования $c$ 
абстрактными обвектами, исследование связей которых позволяет вылвлять новые признаки абстрактных объектов и вводить новые абстракиии» [11, c. 132].

Мысленно невозможно экспериментировать с никак не структурированными точками, которыми, по сути, и являются индивиды реляционных моделей. Такое экспериментирование становится возможным в том случае, если индивид ассоциирован со структурой из функционально связанных элементов. В этом и заключается, с нашей точки зрения, суть генетического метода.

\section{6 Выводы}

C формальной точки зрения, генетический метод построения теорий и генетический стиль мышления имеют лишь историческую ценность. Отличительным признаком генетического метода является использование функциональных языков, которые по своим выразительным возможностям не превосходят, но и не уступают реляционным [12]. Функциональным языкам принадлежит всего лишь исторический приоритет.

С концептуальной точки зрения, разница между генетическим и аксиоматическим подходами значительна. Генетический подход предлагает смотреть на мир как состоящий из вещей, свойств и функциональных связей между вещами. Такой взгляд на мир внутренне динамичен и лучше соответствует нашим интуитивным представлениям. При аксиоматическом, экзистенциальном подходе, мир статичен. В нем ничто не возникает, и ничто не исчезает. Он состоит из уже существующих вещей, находящихся друг с другом в тех или иных отношениях. Даже время в нем статично. Это всего лишь множество точек, находящихся между собой в отношении ранъше-позже.

Современной формой построения генетических теорий является их аксиоматизация в языках с функциональными символами и равенством. Допускается расширение языков одноместными предикатными символами.

В случае использования чистых реляционных языков объект исследования, будучи представлен переменной или в лучшем случае константой, описывается одним или несколькими высказываниями, говорящими о том, в каких отношениях он нахо- 
дится с другими объектами предметной области. Единственным способом анализа свойств такого объекта является логическая дедукция других реляционных высказываний о нем.

В функциональных языках объект исследования представлен термом той или иной степени сложности. Выявление его свойств происходит посредством анализа структуры терма. Рекурсивное сведение свойств объекта к свойствам составляющих его подобъектов является всего лишь частным случаем методов анализа. Неконструктивные способы рассуждений в функциональных языках также являются допустимыми. Они позволяют обнаруживать свойства, возникающие за счет более сложной организации объекта исследования, и лишь частично могут быть объяснены свойствами его подобъектов.

\section{Литература}

[1] Бирюков Б.В. О научных результатах Германа и Роберта Грассманов в свете последующих исследований логики мышления // Грассман Г., Грассман Р. Логика и философия математики. Избранное. М.: Наука, 2008. С. 347-457.

[2] Гилъберт Д. Основания геометрии. ОГИЗ, Москва; Ленинград, 1948. С. 57.

[3] Гилъберт Д. О понятии числа // Основания геометрии. ОГИЗ, Москва; Ленинград, 1948. С. 315-321.

[4] Гилъберт Д., Бернайс П. Основания математики. Логические исчисления и формализация арифметики. М.: Наука, 1982.

[5] Гудстейн Р.Л. Рекурсивная теория чисел // Рекурсивный математический анализ. М.: Наука, 1970.

[6] Клини C.K. Введение в метаматематику. М.: Иностранная литература, 1957.

[7] Крушинский A.A. Генетический тип дедукции - альтернатива традиционно понимаемой дедукции как выводу из аксиом // Логические исследования. Вып. 14. М.: Наука, 2007. С. 176-186.

[8] Мордухай-Болтовский Д.Д. Комментарии // Начала Евклида. Книги I-VI. ОГИЗ, Москва-Ленингад, 1948. С. 221-446.

[9] Начала Евклида. Книги I-VI. ОГИЗ, Москва; Ленинград, 1948.

[10] Смирнов В.A. Генетический метод построения научной теории // Логикофилософские труды В.А. Смирнова. М.: Эдиториал УРСС, 2001. С. 417-437.

[11] Cтепин B.C. Теоретическое знание. М.: Прогресс-Традиция, 2003.

[12] Шалак В.И. Логика функций vs логика отношений // Логические исследования. Вып. 16. М.: Наука. С. 259-271.

[13] Эвклидовыхъ Началъ восемь книгъ // Пер. Ф. Петрушевского. СанктПетербургъ, 1819. 